\title{
POLONESES DE BRASIL Y ARGENTINA UNIDOS EN TIEMPOS DE GUERRA
}

\section{Poloneses do Brasil e da Argentina Unidos em Tempos de Guerra}

\author{
Francisco Pedro ÁLVAREZ \\ Universidad de Buenos Aires \\ alvarezfp@gmail.com \\ https://orcid.org/0000-0002-1956-5189 \\ Claudia Stefanetti KOJROWICZ \\ Universidad de Buenos Aires \\ claudiask@gmail.com \\ https://orcid.org/0000-0002-1990-1277
}

RESUMEN: Durante la Segunda Guerra Mundial, más de dos mil voluntarios de Brasil y la Argentina se unieron a las fuerzas armadas polacas que se organizaron en Gran Bretaña para enfrentar a los Nazis. Esta ponencia se propone iniciar una línea de investigación sobre esta temática para evitar que caiga en el olvido, ya que hasta el presente no se cuenta con memorias publicadas por los protagonistas ni con trabajos académicos que se refieran a los hombres y mujeres de origen polaco que partieron desde América del Sur para incorporarse al Ejército Polaco de entonces. Los archivos son pocos y dispersos, hay que buscar la información con mucho esmero y creatividad porque lamentablemente no hay una institución que se haya ocupado de ellos. Sin embargo, algunos periódicos que acaban de ponerse a disposición de los investigadores en la Biblioteca Nacional de Buenos Aires, permiten hallar nuevas pistas para transitar los caminos que recorrieron los voluntarios. También quisiéramos que este texto sea entendido como una invitación a trabajar colaborativamente entre los argentinos y brasileños para subsanar este vacío enlos estudiosmigratorios referidos a los polacosenAméricadel Sur. PALABRAS CLAVE: Polacos; Brasileños; Argentinos; Voluntarios; Segunda Guerra Mundial; Soldados.

RESUMO: Durante a Segunda Guerra Mundial, mais de dois mil voluntários do Brasil e da Argentina se uniram às forças armadas polonesas que se organizaram na Grã-Bretanha para enfrentar os nazistas. Este artigo pretende iniciar uma linha de investigação sobre esse tema para evitar que caia no esquecimento, visto que até o presente não existem memórias de seus protagonistas publicadas ou trabalhos acadêmicos que se refiram aos homens e mulheres de origem polonesa que partiram da América do Sul para se incorporar 
ao Exército Polonês da época. Os arquivos são poucos e dispersos, tem que se buscar as informações com muito cuidado e criatividade, pois infelizmente ainda não há instituição que trabalhe com eles. No entanto, alguns periódicos acabam de ser colocados à disposição da Biblioteca Nacional de Buenos Aires, permitindo encontrar novas pistas sobre os caminhos percorridos pelos voluntários. Gostaríamos também que esse texto fosse considerado como um convite ao trabalho colaborativo entre argentinos e brasileiros, visando preencher essa lacuna nos estudos migratórios sobre poloneses na América do Sul. PALAVRAS-CHAVE: Poloneses; Brasileiros; Argentinos; Voluntários; Segunda Guerra Mundial; Soldados

Tradução do resumo: Milena Woitovicz Cardoso

\section{FOTO 1 - PARCHE EXHIBIDO LA MUESTRA "LAS ALAS BLINDADAS" DEDICADA AL GENERAL MACZEK Y SUS HOMBRES. MUSEO REAL DE LAS FUERZAS ARMADAS Y DE LA HISTORIA MILITAR. BRUSELAS, FEBRERO 2019}

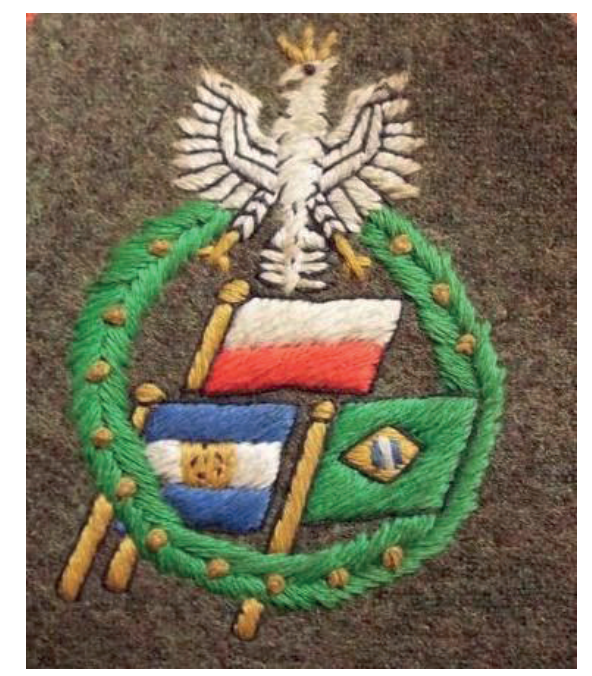

FONTE: F.P. ÁLVAREZ (2019)

Es verdad que la información es escasa y dispersa, pero como ocurre en muchos otros casos, es la prensa periódica quien nos trae respuestas complementarias. Esta ponencia tiene por objetivo dar a conocer la participación de más de 1600 hombres de origen polonés que dejaron la seguridad de vivir en Sudamérica para entrenarse en Escocia y enfrentar al nazismo. Las fuentes consultadas son los Archivos Nacionales de Gran Bretaña, el Instituto Polaco de las Artes y Ciencias de los Estados Unidos (PIASA), 
el Instituto Sikorki de Londres (PISM) y el Museo Real de las Fuerzas Armadas y de la Historia Militar de Bélgica. Muy especialmente nos ha guiado la información que proporciona la Gazeta Polska w Argentynie, editada en Buenos Aires y conservada en la Biblioteca Nacional Mariano Moreno. Un periódico que mantenía relaciones estrechas con la colonia polonesa en Brasil. La comunidad polaca en la Argentina contaba con otras publicaciones periódicas, tal como el Głos Polski, Codzienny Niezależny Kurjer Polski w Argentynie, todas ellas de Buenos Aires, mientras que en Misiones aparecían Orędwonik y Osadnik. En todas ellas se encuentran menciones a las actividades relacionadas con el llamado a las armas, las despedidas y las actuaciones en Europa. Lo distintivo de Gazeta Polska es la referencia a los voluntarios provenientes de Brasil que partieron desde el Río de la Plata.

\section{EL RECLUTAMIENTO}

Desde un principio, el general Władysław Sikorski, primer ministro del Gobierno de Polonia en el Exilio y General en Jefe de las Fuerzas Armadas Polacas durante la Segunda Guerra Mundial, pensó en América donde vivían miles de inmigrantes poloneses como posibilidad para reclutar hombres para su ejército. A pesar de todos sus intentos los resultados no fueron los esperados. El Gobierno en el Exilio asentado aún en Angers, Francia, le pidió a las Legaciones Polacas en Sudamérica que elaboraran un informe pormenorizado de cómo estaba compuesta la población de origen polonés y de las posibilidades de su reclutamiento. Para el mes de noviembre de 1940, el jefe de la Legación Polaca en Buenos Aires, Kazimierz Zdzisław Kurnikowski ${ }^{1}$ había completado su tarea con un detallado estado de la situación del territorio que abarcaba su espacio jurisdiccional compuesto por siete Estados nacionales: Argentina, Bolivia, Chile, Ecuador, Paraguay, Perú y Uruguay. Sólo aquellos cercanos a la costa atlántica contaban con un número verdaderamente amplio de pobladores polacos, a los que distinguió en tres categorías:

- obreros, quienes vivían en las ciudades;

- campesinos, residentes en las colonias y zonas agrícolas, y

- comerciantes y artesanos, mayoritariamente de confesión judía.

\footnotetext{
${ }^{1}$ AKN. Delegación de Polonia en Buenos Aires, 10 de febrero del 1940. No 745/Ar/4. En Adelante, cada vez que lo citemos, estaremos hacienda referencia a este mismo documento.
} 
Kurnikowski se mostraba optimista en cuanto a un posible reclutamiento ya que se habían presentado espontáneamente más de 100 personas. Podemos decir que entre ellas se encontraban algunos inmigrantes que habían llegado recientemente a las colonias Polana y Wanda, en el norte misionero, y algunos ciudadanos judíos. Para febrero de 1940 ya se habían unido 22 hombres al ejército polaco en Francia.

Kurnikowski lamentaba, al igual que aún hoy lo hacemos todos los investigadores, que no existieran datos estadísticos exactos acerca de la cantidad de ciudadanos poloneses que habitaban la región abarcada por la Legación, en la que no eran obligatorios ni el registro ni la renovación de pasaportes. Eso lo llevó a calcular que, aproximadamente, la cantidad de personas polacas que habitaban en la Argentina y en el resto de las repúblicas que caían bajo su jurisdicción era de unas 250 mil almas, de las cuales sólo 50 mil (20\%) serían ciudadanos polacos, el resto serían descendientes o quizás, ucranianos o bielorrusos ${ }^{2}$. Calculó que los hombres entre los 18 y los 45 años serían alrededor de 27.000, de los cuales unos 20.000 estaban casados. De todas maneras estos no eran sus números definitivos ya que pensaba que debía darse una cierta movilidad provocada por la reemigración y por la inmigración ilegal desde Brasil.

A través de los escritos del profesor Ryszard Stemplowski (1976) sabemos que ni bien se conoció la noticia de la invasión Nazi a Polonia, también en Brasil, algunos hombres se presentaron voluntariamente para unirse a su ejército en Francia y que viajaron en el barco M/S Stalowa Wola. Otros 320 hombres siguieron sus pasos entre los años 1940 y 1943.

En su informe, Kurnikowski, había añadido dos reflexiones que nos ayudan a comprender mejor las reacciones de los inmigrantes poloneses frente el ataque que sufrió su patria. Por un lado, hay que tener en cuenta que las oficinas en el extranjero no obligaban a los hombres a unirse al servicio militar, por ejemplo, como condición para conservar su ciudadanía. Esto lo hizo concluir que no habría que esperar una gran diferencia entre la cantidad de personas que se pudieran presentar por una eventual obligación y los que lo harían voluntariamente.

El segundo tema que resalta, seguramente era el más importante al momento de decidir acudir al llamado de Polonia: los gastos. Es preciso comprender que no sólo la inscripción era voluntaria, sino que el mismísimo voluntario debía enfrentar los costos de su participación en las fuerzas militares, es decir, debía correr con todos los gastos

\footnotetext{
${ }^{2}$ Debemos recordar que unos y otros eran sospechados de no ser polacos sino de portar pasaportes de Polonia debido a los cambios de fronteras ocurridos a partir de 1918. Eran vistos como posibles agentes del enemigo.
} 
incluyendo los pasajes transatlánticos. En el caso argentino, recordemos que, los 22 hombres que habían partido inicialmente desde Buenos Aires hacia Francia, lo habían hecho con boletos de repatriación. Estos eran unos pasajes que las empresas marítimas argentinas habían ido acumulando por años, sin embargo por aquellos tiempos habían comenzado a agotarse ${ }^{3}$. Además al no haber muchas personas adineradas en la zona, se hacía difícil pensar que el número de voluntarios pudiera ascender. Los hombres casados y quienes tenían talleres o algún tipo de emprendimiento seguramente no querrían unirse al ejército. Ante esta realidad, Kurnikowski sugirió que se cubrieran los gastos de transporte tanto en el territorio americano como hacia Europa, para incentivar el reclutamiento, y que a las familias se les otorgara algún tipo de compensación económica. Suponía que de esta manera aumentaría significativamente el número de reclutas. Incluso llegó a predecir que serían unos mil hombres. Estaba en lo cierto.

Kurnikowski analizó los costos relacionados con el reclutamiento en la Argentina. El transporte hasta el puerto de la ciudad de Buenos Aires podría trepar hasta los 200 dólares, especialmente cuando se trataba de voluntarios de Chile, Paraguay o Bolivia, mientras que los gastos dentro del país se reducirían a unos 30 dólares.

La espera del barco en Buenos Aires podía extenderse a unos diez días, debido a la poca frecuencia de los trenes que comunicaban al interior con el puerto y a la escasa llegada de barcos con destino a Francia. Los gastos por alimentación, hospedaje y exámenes médicos en Buenos Aires más el transporte transatlántico se calcularon en aproximadamente 197 dólares americanos.

Los primeros voluntarios habían tenido que hacer sus revisaciones médicas en Buenos Aires y todo indicaba que se continuaría con la misma modalidad. Por eso Kurnikowski insistía en que por lo menos se practicara una primera revisación médica en las cercanías del domicilio del interesado, para ahorrar los costos del viaje hacia Buenos Aires en el caso de que el resultado final fuera negativo.

Por entonces, la situación en Europa empeoraba. El Gobierno Polaco en el Exilio que había dejado París para establecerse en Angers en noviembre de 1939, había tenido que movilizarse una vez más. En junio de 1940 tras la caída de París se trasladó a Londres, la ciudad que sería su residencia permanente hasta 1990, cuando el país recuperó la democracia y este gobierno delegó todos sus poderes al nuevo gobierno elegido en Varsovia (DAVIES, 2005, p. 510-517).

\footnotetext{
${ }^{3}$ Los inmigrantes podían acceder a regresar gratis a sus países de origen. Por lo general, el consulado o las sociedades de cada colectividad negociaba con la naviera el regreso de sus compatriotas.
} 
La necesidad de incorporar más hombres al ejército aumentaba dramáticamente, por eso el general Sikorski decidió lanzar una campaña a gran escala en búsqueda de nuevos soldados apelando al patriotismo polaco. En agosto de 1940, se les prometió a los voluntarios en el extranjero que al finalizar la guerra se le otorgarían los mismos derechos y privilegios que recibirían todos los ciudadanos polacos que hubiesen servido en el Ejército Polaco. El Consejo de Ministros les aseguró, entre otras cosas:

a) el derecho a la tierra y a la prioridad de recibirla en el marco de una reforma agraria;

b) prioridad para conseguir trabajo;

c) el derecho a reducciones fiscales extraordinarias y facilidades de crédito. (STEMPLOWSKI, 1976, p. 162).

De todas maneras, los casi 400 voluntarios que habían viajado inicialmente desde Brasil y la Argentina no satisfacía las expectativas del gobierno polaco. Se necesitan muchísimos más y las esperanzas estaban puestas en el Norte. El general Sikorski no sólo había enviado diferentes misiones a Canadá y a los Estados Unidos, sino que él mismo se presentó en ambos países en 1941 y 1942 guiado por el optimismo que le producían los planes de reconstrucción del Ejército Polaco, comenzando por las tropas que habían sido evacuadas de Francia. Calculaba que se sumarían unos tres mil hombres de Gran Bretaña, 170.000 de Canadá y 4.500.000 de polacos de los Estados Unidos, a quienes se les podrían agregar un par de miles de Sudamérica. La realidad era otra, en general y por diferentes razones, esos miles de hombres corrían el riesgo de perder ciertas condiciones de ciudadanía o de refugio. A través de las conversaciones mantenidas con el primer ministro canadiense, Mackenzie King, y con el presidente norteamericano, Roosevelt, buscaba el apoyo de ambos para estimular el reclutamiento de voluntarios. El resultado estuvo muy lejos de los números imaginados. Sólo 700 hombres de ambos países respondieron al llamado de Polonia (KOCHANSKI, 2012).

Durante semanas enteras, hubo funcionarios recorriendo todos los centros poblados por polacos en Brasil y la Argentina, haciendo propaganda de reclutamiento, tanto en sus sociedades como en sus periódicos, para sumar más hombres.

Los voluntarios alistados fueron sometidos a un chequeo médico, en las cercanías de sus domicilios, tal como lo había sugerido Kurnikowski, y si se los encontraba sanos, eran enviados a los puertos de Río de Janeiro, Montevideo o Buenos Aires. En general, los barcos ingleses que los trasladarían al Reino Unidos hacían esas tres paradas. 


\section{EL VIAJE}

Stemplowski (1976) al hablar de Brasil describe la dureza del viaje que el postulante debía realizar entre su hogar y el puerto de salida, sus detalles lo asemejan considerablemente a lo que se vivía en la Argentina.

Las condiciones de viaje eran difíciles, las distancias enormes a duras penas cubiertas por comunicaciones y transportes inadecuados. Basta decir que cuando el Consulado enviaba un cable al postulante, si no se perdía en el camino, podía demorar hasta una semana o dos para llegar a destino. A continuación, el viaje desde un asentamiento en el interior hasta Río de Janeiro tardaba otras dos o cuatro semanas. El área bajo la jurisdicción del consulado en Rio Grande do Sul es comparable con la superficie de Polonia, y en el interior el único medio de transporte era el caballo o un carro tirado por animales, sólo en las ciudades se podía tomar un autobús o, más raramente, un tren para cubrir los siguientes cien kilómetros. (STEMPLOWSKI, 1976, p. 167).

Una vez que el joven llegaba a Río de Janeiro, después de varios días de haber dejado su casa, debía esperar el barco durante varias semanas, a veces, meses. Sin distraerse, pues el transporte amarraba en puerto sólo por 24 horas y no se sabía cuándo se produciría el llamado para abordarlo. Además, se decía que debían mantener un secreto estricto para evitar que los alemanes supieran de su existencia y así impedir un posible ataque nazi en el Atlántico. Y quizás también para evitar algunos inconvenientes con el gobierno de Getulio Vargas que no veía con buenos ojos a estos voluntarios.

Debemos recordar que si bien, en líneas generales, los poloneses compartían el barco con los voluntarios de familias británicas, las condiciones en las que viajaban eran absolutamente diferentes. Los viajes de los jóvenes anglo-argentinos habían sido pagados por sus asociaciones inglesas o escocesas, las que hicieron todo lo posible por garantizarles las mayores comodidades en el traslado.

El viaje transatlántico resultaba tan poco confortable como lo había sido llegar al puerto. Si bien el gobierno polaco había conseguido que Gran Bretaña facilitara el transporte, sus condiciones no eran las ideales, eran simples barcos mercantes adaptados para el transporte de tropas. En una nota enviada por la empresa naviera a cargo del transporte, la Royal Mail Lines, a la Legación Polaca en Río de Janeiro, se detallaban las condiciones de viaje, en julio de 1942, en el Highland Monarch:

1. No hay cabinas para acomodar a estos hombres. Sin embargo, podrán dormir en hamacas que ya han sido usadas con este fin. 
Estas hamacas deberán ser enrolladas cada mañana por los mismos usuarios porque hay que dejar la cubierta despejada.

2. Los hombres deben ir a buscar la comida a la cocina, donde habrá personal suficiente para servirles.

3. La cubierta, los lavatorios, todos los lugares de aseo deben ser cuidados y limpiados por los mismos hombres.

4. El equipaje debe ser lo más pequeño posible, pues no hay lugar en la bodega. (PIASA, Delegación Polaca en Río de Janeiro, 70213 004).

Los voluntarios dormían y comían en las cubiertas, donde también realizaban ejercicios físicos, recibían entrenamiento en la operación de cañones antiaéreos, en tiro y recibían lecciones de idioma inglés.

Franciszek Arciszewski, agregado militar en la Legación Polaca en Río de Janeiro, en una carta enviada a Londres describió crudamente la situación de ellos:

El equipaje de estos hombres era modesto, la mayoría de las veces, fruto de las donaciones que sus compatriotas organizaban en las colonias y centros sociales polacos. Casi todos los voluntarios habían llegado a Río de Janeiro desde el interior con un solo traje bajo el cual a menudo no llevaban ropa interior, sus zapatos generalmente estaban rotos. En general se compraron por primera vez pantalones de lino, chaquetas de abrigo, botas, ropa interior y una boina. Muy raramente contaban con dinero propio y lo que se les pagaba en Río de Janeiro apenas podía alcanzar para comprar un par de paquetes de cigarrillos. (...). La mayoría absoluta de los voluntarios era gente extremadamente pobre que no había ganado nada emigrando a Brasil (PIASA, Delegación Polaca en Río de Janeiro, 70213 006).

Las misiones en Brasil y la Argentina produjeron mejores resultados que las extensas campañas organizadas en América del Norte. Como hemos visto, sólo se pudieron reclutar unos 700 hombres a pesar de la gran campaña realizada. Cuando se dan los números argentinos se debe tener en cuenta que en muchos casos, no siempre, se incluyen algunos voluntarios provenientes de Bolivia, Chile, y Uruguay sin hacer las aclaraciones pertinentes. Deberíamos preguntarnos por qué no hay datos de Paraguay. Al realizar varias entrevistas a inmigrantes polacos de este país ${ }^{4}$, hemos recibido la misma respuesta y es que "eran muchas las personas con deseos de unirse a las filas polacas, pero

\footnotetext{
${ }^{4}$ Desde el año 2000 realizamos entrevistas a inmigrantes polacos dentro del marco del Proyecto "Las Huellas Polacas en la RA", en el Museo Roca de la Ciudad de Buenos Aires. Se lo puede consultar en su sitio web: <www.aguilablanca.com.ar>
} 
que el encargado de tal reclutamiento falleció y nadie lo reemplazó, y así sin más, todo quedó en la nada." Esta es una información que necesita de cierto respaldo documental que aún no hemos hallado. También es posible que algunos voluntarios polacos asentados en Paraguay hayan salido de Buenos Aires con domicilio argentino. Por regla general, los pocos registros con los que se cuenta, especialmente los que conserva el Instituto Sikorki de Londres, no siempre establecen el domicilio de residencia efectiva del voluntario. Los datos filiatorios estaban orientados a demostrar su nacionalidad polaca más que su lugar de residencia.

Como ya vimos, un poco menos de 400 voluntarios dejó Brasil desde mediados de 1940 hasta 1943, año en que las postulaciones prácticamente desaparecieron. Para entonces, habían partido unos 1200 hombres y 8 mujeres desde Buenos Aires. ${ }^{5}$ Este es el momento en que debemos hacer un alto y comenzar a diferenciar a los grupos provenientes de Brasil y de la Argentina. Así como las condiciones de reclutamiento y de viaje pudieron ser similares, las normas para alistarse fueron mutando de acuerdo a los cambios políticos en cada país.

\section{NORMAS BRASILEÑAS}

En Brasil, en primer lugar, únicamente estaban autorizados a alistarse los ciudadanos polacos, lo cual excluía a las personas nacidas en Brasil aunque sus dos padres sí lo fueran. Hubo una disposición que les permitía a los ciudadanos brasileños de origen polaco aplicar al ejército (no incluía a los reservistas del ejército brasileño) pero el procedimiento era muy lento, ya que cada caso necesitaba la aprobación del presidente Vargas y lo cierto es que nadie obtuvo ese permiso, aunque muchos lo intentaron (PIASA. Delegación Polaca en Río de Janeiro, 70213 007).

La situación política interna influyó mucho más en Brasil que en la Argentina a la hora de los alistamientos voluntarios. Por lo general, en la Argentina las autoridades miraban hacia otro lado y dejaban hacer, pero en Brasil los gobiernos federales actuaban intensamente en contra de la propaganda de las autoridades polacas e intimidaban a los voluntarios. Uno de los objetivos más importantes de las políticas de Vargas era acelerar el proceso de integración nacional en este enorme y culturalmente heterogéneo país. En este contexto las actividades políticas y culturales de las diferentes comunidades de

\footnotetext{
${ }^{5}$ Las cifras, estimadas, son fruto del cruce de documentos conservados en la Asociación de Ex Combatientes Polacos en la República Argentina y de los datos disponibles en el Instituto Sikorski de Londres.
} 
inmigrantes fueron restringidas drásticamente en 1938, cuando se cerraron escuelas, sociedades y periódicos para promover la asimilación y la lengua portuguesa. Los decretos de "nacionalización", como los llamaban los polaco-brasileños habían sido estrictamente observados e intensificados durante la guerra. La Legación Polaca tuvo que hacer grandes esfuerzos para que Vargas autorizara el reclutamiento. Recién pudieron tener una campaña abierta en 1943. De todas maneras, los obstáculos fueron incontables y el reclutamiento se vio impedido de variadas formas (STEMPLOWSKI, 1976, p.165).

En ese mismo año 1943, le Legación Polaca hizo un llamado a las mujeres para que se unieran a las filas como auxiliares en diferentes actividades para que los hombres que ocupaban esos puestos, como los telegrafistas, pudieran ir al frente. No se sabe de ninguna mujer de origen polaco que haya partido de Brasil para unirse a este cuerpo. ${ }^{6}$

Podríamos decir que en Brasil hubo diversos momentos de reclutamiento, no sólo por las políticas implementadas por Polonia sino por el mismo Estado brasileño. Principalmente podemos distinguir un primer momento que comienza en junio de 1941, cuando los polacos hacen un llamado formal a sus hombres, hasta diciembre de 1942. Durante ese tiempo, la cuestión de las visas de entrada a Brasil no estaba completamente regulada. Cualquier extranjero podía recibir una visa de salida si presentaba sus documentos de ingreso legal al Brasil previo a 1938. Con posterioridad a esa fecha, debía tener un Carnet de Identidad de Extranjero conocida como "Modelo 19" que fue instituido en forma obligatoria en 1938, durante el gobierno de Getulio Vargas. A través del decreto 3010 de agosto de ese año se estableció que todos los extranjeros menores de 60 años estaban obligados a registrarse en la dependencia policial de su lugar de residencia, donde se les otorgaría un certificado que acreditaba su residencia temporal o permanente en el territorio brasileño. Este es el documento que otorgaba la Policía Federal y que por décadas fue conocido como Modelo 19 (BRASIL, 1938).

Las notas que intercambiaron los funcionarios polacos en Río de Janeiro o Curitiba, con los delegados ingleses en Brasil o Londres están plagadas de referencias a la necesidad de darles pasaportes polacos a estos hombres que se presentaban voluntariamente para formar parte del Ejército, quienes decían haber perdido sus documentos originales. La mayoría, si no todos, dejaron América con pasaportes recién hechos (PIASA. Delegación Polaca en Río de Janeiro, 70213 007).

\footnotetext{
${ }^{6}$ Puede verse dicho llamado en todas las portadas del mes de mayo de 1943 del diario Glos Polski de Buenos Aires.
} 


\section{LA VÍA ARGENTINA}

Todas las trabas que imponía el gobierno federal, no impidieron que más de una docena de jóvenes dejaran Brasil ilegalmente rumbo a Europa, algunos argentinos también lo hicieron. Hubo cuatro brasileños que se hicieron famosos gracias a un diario polaco de Buenos Aires: la Gazeta Polska w Argetyna, un periódico que tenía estrechas relaciones con Curitiba y habitualmente publicaba noticias de la región paranaense. Los voluntarios eran Antoni Ostrowski, Juliusz Czaplinski, Stanislaw Jeziorowski y Lucian Wilke (Gazeta Polska w Argentyna, 1941). Dejaron Buenos Aires en el transporte número IV, el 5 de septiembre de 1941, fueron registrados como polacos de Argentina, tal como había ocurrido con los dos sobrinos de Ostrowski unos meses antes. Este es un ejemplo más de lo difícil que es establecer el origen concreto de los voluntarios de Sudamérica. De hecho, la verdad es que eran ciudadanos brasileños, nietos de polacos.

\section{FOTO 2 - RECEPCIÓN A LOS VOLUNTARIOS BRASILEÑOS ORGANIZADA POR LA GAZETA POLSKA W ARGENTYNA 04 SET.1941}

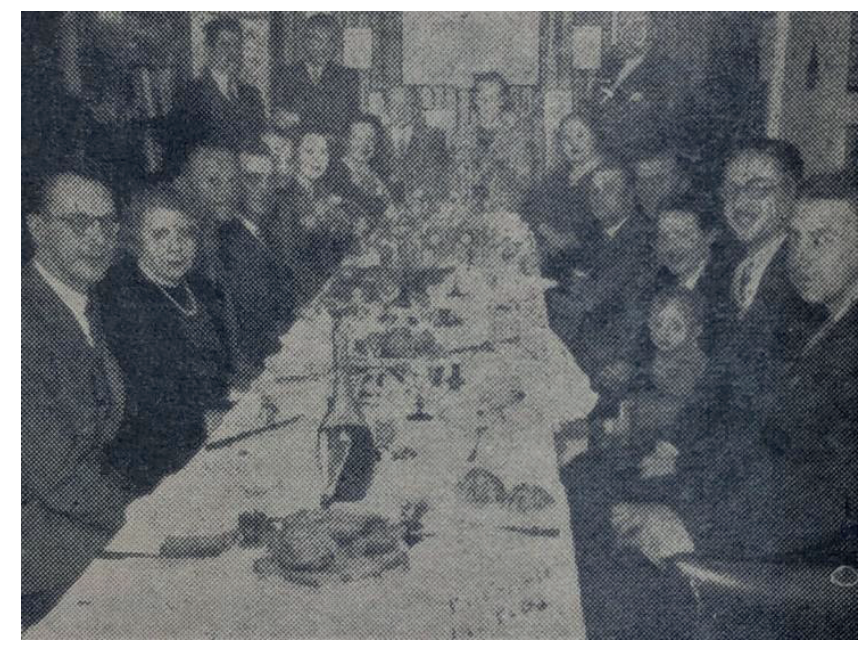

FONTE: GAZETA POLSKA W ARGENTYNA (1941)

Aquellos cuatro jóvenes fueron agasajados en todas las fiestas que la comunidad polaca organizaba para despedir a sus hijos, se hacían comidas, se reunía dinero, ropa, cigarrillos, jabones. Todo lo que pudiera serles útil a quienes se iban a pelear por Polonia. ${ }^{7}$

\footnotetext{
${ }^{7}$ El diario Głos Polski publicó los anuncios y las fotos de gran parte de estas reuniones. Desde julio de 1941 hasta finalizada la guerra en 1945, aparecían reportajes, fotos y notas que daban
} 
Esta manera de agasajar a los voluntarios fue puesta de relieve por Arciszewski para hacer notar lo que faltaba en Brasil.

\begin{abstract}
Cuando un voluntario sale desde la Argentina da discursos y es acompañado al puerto por una multitud de compatriotas. Un voluntario brasileño se va subrepticiamente, conspirando contra el propósito de su partida. Esto no debilita el espíritu de los pensadores valientes, pero tampoco alienta ni motiva a los más indiferentes o de sangre fría (PIASA, Delegación Polaca en Río de Janeiro, 70213 004).
\end{abstract}

Es cierto que la Gazeta Polska registró la mayor parte de los eventos relacionados con los voluntarios en la Argentina: fiestas, despedidas, visitas a la redacción del diario, algunas de las partidas. Pero también es verdad que las realidades políticas al interior de ambos países eran muy diferentes. Las políticas de Vargas eran abiertamente contrarias a la convocatoria de Polonia mientras que el gobierno argentino había adoptado una actitud de neutralidad amistosa.

Antoni Ostrowski y sus amigos tuvieron su primer entrenamiento en Escocia, como el de todos los voluntarios llegados desde el Río de la Plata, pero sólo algunos pocos elegidos pudieron unirse a la Fuerza Aérea. Ostrowski ingresó al Escuadrón 305 Ziemia Wielkopolska ${ }^{8}$. Participó en cuatro misiones aéreas desde el 6 de septiembre de 1942 hasta su muerte el 23 del mismo mes, cuando su avión fue derribado cerca de Brest, Francia. Su cuerpo descansa en el cementerio de Quiberon, en la región de Bretaña. ${ }^{9}$

Antoni no fue el único de la familia en dejar Brasil para ingresar a la Fuerza Aérea, sus sobrinos Marceli y Edmund recorrieron el mismo camino que los llevaría a unirse a la aviación. Paradójicamente, los Ostrowski habían llegado al puerto de Santos en 1907 desde la misma Gran Bretaña. Con el tiempo, fueron cambiando sus lugares de residencia dentro de la región paranaese, donde ya había un gran número de colonos polacos. En la década de 1920 los Ostrowski se dedicaban a la industria forestal, construían durmientes, carros, casas y hasta una escuela en Marumbí que fue visitada por Władysław Raczkiewicz, por entonces representante del Senado de Polonia, y más tarde Presidente del Gobierno Polaco en el Exilio.

cuenta de estos eventos. Como así también de lo que ocurría con los jóvenes en Europa. No hay menciones a polacos de Brasil, sino de las asociaciones de la República Argentina.

8 "Ziemia Wielkopolska" o Tierra de la Gran Polonia, era una unidad de bombarderos polacos que luchó junto a la Royal Air Force y operaba desde bases aéreas en el Reino Unido

${ }^{9}<$ https://listakrzystka.pl/> Los datos de los tres Ostrowski pueden consultarse en este sitio 
Marceli y Edmund Ostrowski estudiaban en la Universidad de Curitiba y ya incursionaban en el pilotaje cuando estalló la Segunda Guerra Mundial. Decidieron dejar los estudios e ir juntos a defender la patria de sus mayores. Eran ciudadanos brasileños, no podían alistarse en su país. Entonces organizaron un plan de fuga: irían a Buenos Aires como turistas, en un simple viaje de placer. Así lo hicieron ellos, de la misma manera que unos meses más tarde lo hizo Antonio con sus amigos, como ya lo hemos visto. Desde allí, en julio de 1941, navegaron en el Highland Princess, nave inglesa, a Gran Bretaña. Marceli estaba calificado para el entrenamiento de vuelo como tirador a bordo, después de entrenar en Blackpool y en Stormy Down, fue asignado al Escuadrón de Bombarderos 301 en Bramcote. Tuvo su primer vuelo de combate el 30 de mayo de 1942, el día en que cumplía 22 años. Estaba volando en un Wellington con el objetivo de bombardear Colonia. Fue el inicio de una larga vida ligada al aire y a los aviones. Llegó el final de la guerra, se casó con una mujer polaca miembro la WAAF (Women's Auxiliary Air Force), el cuerpo femenino auxiliar de la Fuerza Aérea. El mismo cuerpo al que se unieron las voluntarias que partieron del Puerto de Buenos Aires en 1943.

Marceli decidió regresar a Brasil para establecerse en Curitiba con su esposa. Sería el único de los tres Ostrowski que regresaría. Como vimos, su tío murió en Francia y su hermano Edmund desafortunadamente encontró la muerte el 22 de febrero de 1944 volando un Mosquito, en Lasham. Su tumba está en el cementerio de Newark, Inglaterra. Sólo tenía 21 años. Tanto Edmund como Antoni pertenecieron al Escuadrón 305.

Lamentablemente la esposa de Marceli no se adaptó al clima de Curitiba y decidieron mudarse a Canadá. Desde 1956, ha estado activo en la Asociación de Aviadores de Polonia (SLP) y en la Asociación de Ex Combatientes Polacos en Canadá (SPK) de la que ha sido Presidente. También integra un equipo que se propuso construir en Toronto un monumento dedicado a la memoria de los pilotos polacos que participaron en la Segunda Guerra Mundial en Gran Bretaña.

A fines de noviembre de 2019, unos días antes del Primer Encuentro de Estudios Poloneses en Curitiba, el teniente coronel Marceli Ostrowski había sido el invitado de honor en una celebración organizada en el Consulado Polaco en Toronto. Fue una reunión llena de memorias de una vida extraordinaria, que lo encontró rodeado por su hijo, sus nietos y bisnietos, por los recuerdos de los árboles de Polonia y Canadá, antecedidos por el bosque brasileño. ${ }^{10}$ Ostrowski no habló de él sino del valor que tuvieron aquellos

\footnotetext{
${ }^{10}$ Por esta razón, este hilado de árboles, es que a su biografía la tituló Wyżej niż piniory (Más alto que los pinos).
} 
hombres comunes al dar la vida por la libertad de todos. Le cantaron un "dwieście lat" ", porque los cien años, "Sto lat", los cumpliría el 20 de mayo y no era cuestión de desearle que sólo viviera unos meses (KUMOR, 2019).

Marceli Ostrowski será condecorado por el Presidente de la República de Polonia, Andrzej Duda, con la Orden del Águila Blanca. Actualmente ningún aviador polaco vivo es titular de esta importantísima orden (MAŃKOWSKI, 2020).

\section{Las reglas brasileñas después de 1943}

A partir del $1^{\circ}$ de enero de 1943, el gobierno de Vargas cambió las reglas de juego. A partir de entonces, sólo podrían conseguir una visa de salida quienes tuvieran en su poder una certificación Modelo 19. Todos los demás documentos, válidos hasta entonces, perdían su legalidad para pedir una visa de salida. Esto implicó que todos los extranjeros que vivían en Brasil deberían tramitar esta cédula de identidad a partir del $1^{\circ}$ de enero de 1943.

Este cambio no sólo influía en la posibilidad de salir de Brasil, sino de regresar. A esto se le sumó un tema prácticamente nuevo: las asignaciones familiares para los voluntarios casados. En la primera etapa sólo se habían aceptado voluntarios solteros, más adelante hubo un cierto número de hombres casados a los cuales se les debía otorgar una pensión para la familia. Esto complicaba el otorgamiento de visas de salida, se las demoraban varias semanas. Todo desalentaba la partida.

Para que todos los trámites fueran realizados en un tiempo acorde a las necesidades, los funcionarios polacos en Río de Janeiro tuvieron que trabajar duramente para lograr la emisión de las cédulas de identidad que luego podrían ser renovadas en la Embajada de Brasil en Londres.

Los obstáculos con los que se encontraban los potenciales voluntarios eran variados, no sólo los impedimentos gubernamentales iban contra las posibilidades de viajar. No conocer los tiempos de llegada de los barcos hacía que tramitar las visas fuera un acto a ciegas, caro y de difícil concreción. Por eso algunos hombres decidieron salir por Buenos Aires o de manera no oficial. De todas maneras, en 1943 se detuvo el flujo de voluntarios desde Brasil.

\footnotetext{
${ }^{11}$ dwieście lat es una frase que no existe, que inventaron en esta ocasión para desearle que viva "doscientos años", en lugar de cien años o "sto lat", que es la canción con la cual los polacos expresan buenos deseos y larga vida. Ostrowski pudo celebrar sus cien años en las condiciones sociales que el Covid 19 le ha permitido.
} 
Toda América le dio a Polonia un total de 2.319 voluntarios. De ellos, 392 se unieron a los escuadrones que sirvieron a la Royal Air Force y 92 lo hicieron en la Marina polaca. La mayor parte de los polacos de Argentina y Brasil fueron enviados a Escocia para unirse al General Maczek y su Primera División Blindada. Allí, en un principio, trabajaron en mantenimiento y como conductores. Luego fueron parte de las batallas de Normandía, del Bolsillo de Falaise, de la liberación de Bélgica y Holanda. ${ }^{12}$

Los polacos desembarcaron en Normandía durante los primeros días de agosto de 1944. Tuvieron un bautismo de fuego devastador. Después de reponer las pérdidas, el 14 de agosto lanzaron un segundo ataque, a los seis días, que logró la ocupación de Falaise. Los soldados de Maczek debían cerrar el paso de los alemanes y para ello el 19 de agosto tomaron Chambois y las colinas de Mont Ormel. Durante los siguientes dos días, reprimieron los intensos ataques de las fuerzas alemanas. Fue una de las batallas más duras y feroces que se libraron en esta guerra. No hubo auxilio para los heridos, ni reabastecimientos de suministros y municiones. A pesar de esto, los polacos lograron mantener la posición. La mayor parte de las bajas producidas entre los voluntarios sudamericanos se dieron en estas tierras que hasta hoy los cobijan en diferentes cementerios de Normandía. Más de treinta soldados que habían partido de Buenos Aires, descansan allí. Otros tantos quedaron lisiados, sin posibilidades de retorno debido a las disposiciones de las leyes argentinas de inmigración. Al finalizar la guerra se establecieron en Gran Bretaña a la espera de una autorización para regresar que jamás les fue dada.

Maczek y sus hombres, después de las acciones en Normandía, continuaron la persecución del enemigo. Liberaron Abbeville y St. Omer, para dirigire hacia Bélgica y liberar Ypres, Tielt, Gante y otras pequeñas ciudades en el camino. En septiembre de 1944 , los polacos cruzaron la frontera entre Bélgica y Holanda. A finales de octubre la $1^{\text {a }}$ División Blindada tuvo el más importante de todos sus logros: liberó a la ciudad de Breda que se llenó de alegría, flores, fiestas y agradecimientos que siguen presentes hasta hoy en sus monumentos, museos y plazas. La guerra continuaba y en abril de 1945 cruzaron las fronteras alemanas. Liberaron un campo para prisioneros políticos holandeses cerca de Westerbork y el de Oberlangen, que tenía entre sus prisioneros a 1.728 mujeres que habían participado en el Levantamiento de Varsovia. Varias de las jóvenes se casaron con sus liberadores y años más tarde, algunos de estos matrimonios se establecieron en la

${ }^{12}$ Cifras que se desprenden de los archivos consultados en el Instituto Sikorki de Londres. Son números provisorios, ya que se trata del primer acercamiento al tema. Los soldados fueron registrados por lugar de nacimiento, no de residencia. Incluso en algunos casos sólo se consignó el apellido completo y la incial de su nombre de pila. 
Argentina. (McGILVRAY, 2013, p. 268)

La guerra estaba llegando a su fin. Después de los feroces combates, el 5 de mayo se dio la capitulación de Alemania. Los voluntarios americanos que estuvieron en todos estos teatros de operaciones ya podían volver a casa, la guerra había terminado.

\section{ELLAS TAMBIÉN FUERON VOLUNTARIAS}

En 1943, el Gobierno Polaco en el Exilio hizo un llamado para que las mujeres se unieran a las fuerzas armadas polacas como cuerpo auxiliar. El barco Rangitiki saldría del puerto de Buenos Aires con el décimo octavo transporte de voluntarios, en él por primera y única vez se incluirían mujeres. Era el mes de agosto de 1943, viajaban ocho mujeres. Sabemos muy poco sobre ellas. Era un grupo compuesto por jóvenes veinteañeras polacas residentes en la Argentina, Chile y Uruguay.

Dos se casaron con polacos en Gran Bretaña y nunca regresaron. Eran de Misiones y una de ellas era hermana de un joven voluntario que había partido hacia Escocia en 1941. Allí se reencontraron, pero no volvieron juntos, él volvió a su pueblo misionero mientras ella comenzó una nueva vida en Gales.

En cambio, otra mujer, la más conocida, regresó a la casa familiar, en la ciudad de Berisso, en el sur de Buenos Aires. Su padre, era el escritor Florian Czarnyszewicz, un miembro muy activo en la vida social y cultural de las Unión Polaca. Se cuenta que sus libros, caracterizados por las largas descripciones de Polonia oriental, eran leídos por los soldados del general Maczek, en Escocia, gracias a que los había llevado su hija. Władysława, quien trabajaba con ellos como tipógrafa. ${ }^{13}$

En el barco también viajaba una familia polaca de Uruguay, compuesta por el matrimonio y dos hijas. Los pocos datos que se obtuvieron dicen que volvieron los cuatro, es decir, cinco ya que una de las jóvenes se había casado con un polaco en Inglaterra.

Este sí es un tema totalmente ignorado en los estudios migratorios. Ellas no están incluidas en ningún listado de voluntarios a los que se puede acceder. Sus nombres figuran en los libros de pasajeros que se pueden consultar en los Archivos Nacionales de Gran Bretaña, pero no están incluidas en los demás archivos militares con los que hemos podido trabajar. Estas mujeres, por lo general realizaron trabajos de oficina, de telecomunicaciones o de conductoras de diferentes tipos de vehículos para liberar a los hombres de esos puestos para que pudieran ir a los diversos frentes.

${ }^{13}$ Los datos sobre estas mujeres fueron obtenidos en diferentes entrevistas con sus familiares y en las bases de datos de CEMLA (Centro de Estudios Migratorios Latinoamericanos). 


\section{Los hermanos}

Otro caso que quedó absolutamente olvidado en las narraciones argentinas, por lo menos, pero que en su momento fue nota en muchos diarios argentinos, polacos e ingleses fue el de los hermanos Widła. Hecho que hizo que el mismísimo General Sikorski se emocionara y le escribiera al padre de los jóvenes.

Eran cuatro hermanos que se alistaron como voluntarios e hicieron el primer entrenamiento con si la 16ta Brigada de Tanques del Primer Cuerpo Polaco en Escocia, en la zona de Kelso. Allí hacían el entrenamiento de manera conjunta los polacos provenientes de Brasil, Argentina y Uruguay. Quizás pueda haber habido polacos de Paraguay y Chile. Estaban reunidos bajo la categoría de "Voluntarios Sudamericanos", siendo los más numerosos los de Brasil y Argentina.

\section{FOTO 3 - KAZIMIERZ (24), WŁADYSŁAW (21), JÓZEF (20) Y MIECZYSŁAW (19) WIDŁA}

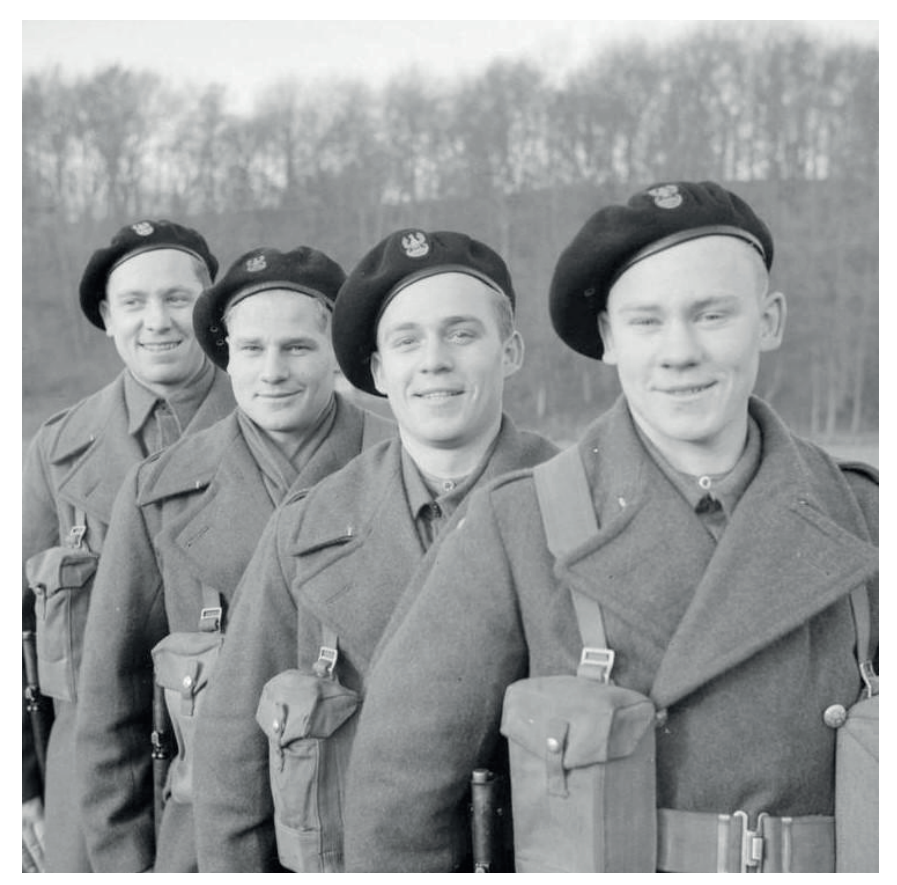

FONTE: IWM, Londres

Estos jóvenes que fueron promocionados como ejemplo de patriotismo eran hijos de una familia de agricultores polacos establecidos en Oberá, Misiones. En total eran siete hijos varones, los dos menores se quedaron en la casa de sus padres por razones de 
edad. El mayor, nunca había estado en la Argentina, había permanecido en Polonia y fue deportado a Siberia cuando estalló la guerra. Los cuatro hermanos fueron saludados por el presidente en el exilio Wladisław Raczkiewicz durante su visita a las tropas formadas por voluntarios de Sudamérica. El Imperial War Museum de Londres conserva una muy interesante colección de ese momento, en la que además se ven soldados con estandartes polacos de Porto Alegre.

\section{FOTO 4 - POLACOS DE PORTO ALEGRE.}

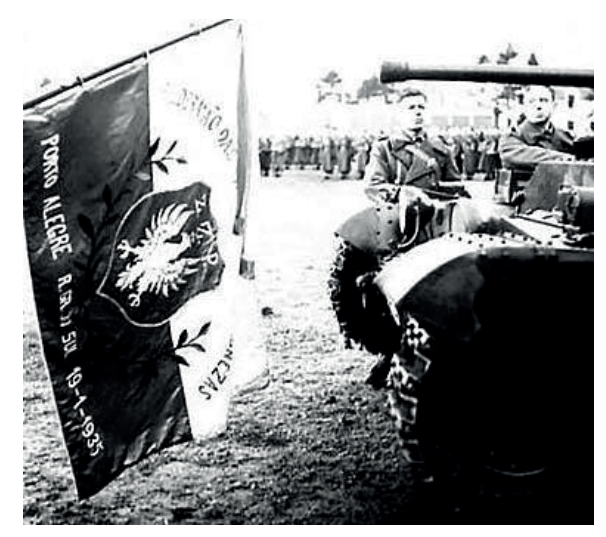

FONTE: IWM, Londres

El general Sikorski supo de la presencia de estos jóvenes y le escribió a su padre una carta que le fue enviada a través del encargado de la Legación en Buenos Aires. El 19 de diciembre de 1941 el General le decía al señor Tadeusz Widła:

Le envío mi mayor expresión de reconocimiento, profundamente conmocionado con el más hermoso ejemplo y modelo de sentimiento nacional y también de deberes para con la República de Polonia. Estoy convencido, que sus cuatro hijos entregados al servicio a la patria desempeñarán dignamente su deber militar (Osadnik, 1942).

El jefe de la Legación, el señor Kurnikowski, también felicitaba cordialmente al señor Widła por el honor que poseía y junto a los saludos le enviaba el deseo de que esos hijos que habían sido criados tan hermosamente, con un espíritu nacional como soldados vencedores de la República, regresaran pronto a la patria liberada.

Los cuatro hermanos regresaron a Misiones en 1946. Nunca volvieron a Polonia, había quedado detrás de la Cortina de Hierro. Años más tarde, el décimo hermano que había sido prisionero en el Gulag soviético se unió a la familia en la Argentina. 
FOTO 5 - BENDICIÓN DEL ESTANDARTE POLACO DE PORTO ALEGRE, ESCOCIA.

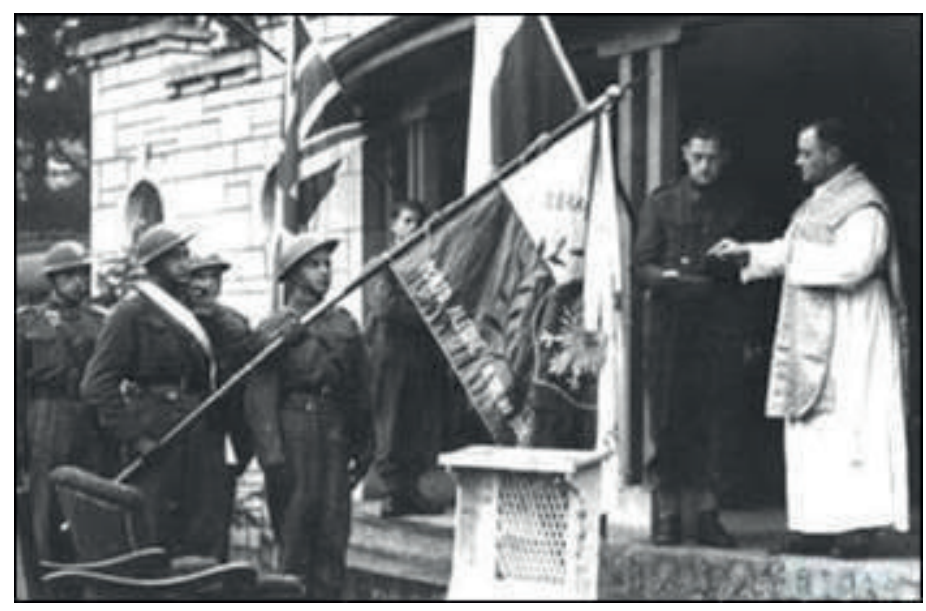

FONTE: IWM, Londres

\section{Los que no pudieron reingresar}

Una vez que finalizó la Segunda Guerra Mundial, la mayoría de los voluntarios regresó a sus países de origen. Otros, hicieron de Gran Bretaña su nueva patria al formar una familia con mujeres locales. Algunos de ellos encontraron un gran impedimento al regresar junto con más de mil compañeros de armas: la guerra les había dejado heridas insanables, habían perdido alguno de sus miembros o más de uno. Primero fueron atendidos en Escocia y una vez que estuvieron lo suficientemente repuestos se les permitió embarcar junto con otros voluntarios y con otros polacos que venían por primera vez América.

El barco Highland Monarch había salido de Inglaterra el 26 de septiembre de 1946 transportando a la mayor parte de aquellos los voluntarios que habían dejado la Argentina cinco años antes. El barco llegó al puerto de Buenos Aires el 16 de octubre. La ley argentina es clara: le abre las puertas a todas las personas que quieran habitar el suelo argentino, siempre y cuando trabajen. Se entiende que para trabajar una persona no debe tener problemas de salud. ¿Cuál fue el destino de los malheridos? Las Actas de Inspección marítima son claras: había 18 hombres de nacionalidad polaca que no eran aptos para trabajar. Esos hombres deberían regresar al puerto de origen. Fue inútil todo tipo de peticiones y ruegos que incluyeron cartas a la esposa del Presidente de la Nación: Eva Perón, una mujer que generalmente intercedía en estos casos. No hubo ningún tipo de contemplación para con ellos. Pasaron 19 días en un hotel cercano al puerto y nuevamente 
fueron embarcados hacia Escocia. Allí tres hombres tuvieron la suerte de mejorar su salud, los otros no. Los primeros volvieron a la Argentina, el resto tuvo las puertas cerradas definitivamente. Durante más de dos años el gobierno británico desarrolló todo tipo de actividades tendientes a obtener el permiso para ellos, prometió darles pensiones vitalicias y todo lo que necesitaran. Fue inútil. Nunca más estuvieron en las costas del Río de la Plata (UK Parliament, 1947).

\section{PALABRAS FINALES}

Al producirse la invasión de Polonia en septiembre de 1939, tanto en la Argentina como en Brasil, la respuesta no se hizo esperar. En un principio, partieron por su cuenta voluntarios de ambos países, sin necesidad de ser llamados oficialmente. Fue mucho mayor el número de hombres que salieron de Brasil, se calcula que fueron unos 360 voluntarios hasta mediados de 1940, mientras que desde la Argentina sólo hay registro de alrededor de 20. Pero a partir de 1941, cuando el Gobierno Polaco en el Exilio logró organizar sus políticas de reclutamiento, se encontró con cambios en la política exterior de Brasil y de la Argentina. Así, fue como los polacos de Brasil se enfrentaron a diversos impedimentos que los condujeron a no alistarse en el Ejército Polaco desde su lugar de residencia y debieron viajar a Buenos Aires. Mientras tanto, el gobierno argentino si bien no apoyaba estos alistamientos, tampoco los impedía. Desde la Argentina partieron diecinueve contingentes de voluntarios entre el 17 de julio de 1941 y el 4 de enero de 1944. Los barcos no siempre consignaron claramente el lugar de procedencia de los voluntarios en sus listados, teniendo en cuenta esa realidad, podemos decir que más de 1200 hombres residentes en la Argentina fueron parte de esos contingentes. Pero para entonces las políticas brasileñas impidieron que creciera el número de voluntarios que quisieran sumarse a estos barcos en su paso por los puertos de Río de Janeiro o de Santos.

Además, en Brasil, el principal slogan utilizado para atraer hombres que quisieran unirse al cuerpo de voluntarios había sido la promesa de un retorno a Polonia con muchísimos beneficios, pero al finalizar la guerra esta propuesta ya no era posible y los anuncios hechos por el Gobierno en el Exilio, pasaron a carecer de toda validez. La mayor parte de los voluntarios regresaron a sus respectivos países, algunos se quedaron en donde los encontró el cese de las hostilidades y una esposa, como Jan Stangryczuk de Oberá, amigo de los hermanos Widła, y quienes buscaron un nuevo horizonte, como Marceli Ostrowski.

Para finalizar queremos recordar especialmente a estos dos hombres de la Aviación 
porque aún viven. Porque siguen siendo esos jóvenes con menos de 20 años que fueron a pelear contra el nazismo. Son los únicos voluntarios que todavía nos pueden contar lo que significó aquel esfuerzo enorme. No sólo en términos de documentos oficiales, sino que al contar en primera persona sus experiencias pueden hacer que entendamos por qué se deja la comodidad de una casa y se pone un uniforme para combatir a miles de kilómetros. Si estos hombres no estuvieran para contarnos sus experiencias es posible que esta historia de voluntarios se hubiese perdido para siempre. Este texto es sólo un comienzo que espera abrir caminos de investigación conjuntos entre los polacos de América del Sur. 


\section{REFERENCIAS:}

BRASIL. Decreto $\mathrm{n}^{\circ}$ 3.010, de 20 de agosto de 1938. Regulamenta o dercreto-lei n. 406, de 4 de maio de 1938, que dispõe sobre a entrada de estrangeiros no território nacional. Diário Oficial da União, Rio de Janeiro, seção 1, p. 16792, 22 ago. 1938. Disponible en: $<$ https://www2.camara.leg.br/legin/fed/decret/1930-1939/decreto-3010-20-agosto-1938348850-publicacaooriginal-1-pe.html>. Acesso em: 20 ago. 2020.

DAVIES, Norman. God's Playground. A history of Poland. Oxford: Oxford University Press, 2005.

DAVIES, Norman. Europa en guerra 1939-1945. Tradução de Amado Diéguez. Barcelona: Planeta, 2008.

Gazeta Polska w Argentynie, Buenos Aires, 18 set. 1941

JAROSZYNSKA-KIRCHMANN, Anna D. The Exile Mission: The Polish Political Diaspora and Polish Americans, 1939-1956 (Polish and Polish American Studies). Athens: Ohio University Press, 2004.

KIENIEWICZ, Jan. Historia de Polonia. Ciudad de México: Fondo de Cultura Económica, 2001.

KOCHANSKI, Halik. The Eagle Unbowed: Poland and the Poles in the Second World. Cambridge: Harvard University Press, 2012

KRZYSTEK, Tadeusz; KRZYSTEK, Anna. Listakrzystka.pl - Personel Polskich Sit Powietrznych $w$ Wielkiej Brytanii 1940-1947. Disponible en: <https://listakrzystka.pl/> Acesso em: 20 ago. 2020.

KUMOR, Andrzej. Ppłk Marceli Ostrowski gościem jesiennego spotkania Stowarzyszenia Inżynierów Polskich w Kanadzie. Goniec, Mississauga, 27 nov. 2019. Disponible en: $<$ https://www.goniec.net/2019/11/27/pplk-marceli-ostrowski-gosciem-jesiennegospotkania-stowarzyszenia-inzynierow-polskich-w-kanadzie/>. Acesso em: 20 ago. 2020.

McGILVRAY, Evan. Man of Steel and Honour: General Stanislaw Maczek: Soldier of Poland, Commander of the 1st Polish Armoured Division in North-West Europe 1944-45. Warwick: Helion and Company, 2013. (Helion Studies in Military History Book 18).

MAŃKOWSKI, Leszek. Ostrowski ma 100 lat! Rada Seniorów Lotnictwa, Gdów, 12 jun. 2020. Disponible en: <https://www.rslap.org/post/marceli-ostrowski-ma-100-lat $>$. Acesso em: 20 ago. 2020. 
Osadnik, Posadas, 31 jan. 1942.

OSTROWSKI, Marceli. Wyżej niż piniory. Varsovia: Soony Project, 2012.

POTASH, Robert A. El ejército y la politica en la Argentina 1928-1945. Buenos Aires: Editorial Sudamericana, 1981.

STEMPLOWSKI, Ryszard. Enlistment in Brazil to the Polish Armed Forces, 1940-1944. En: Polish Western Affairs, v. 17, n.1-2, Poznan: Institytut Zachodni, 1976.

UK Parliament. Disabled Poles (Argentine). 17 jun. 1947, v. 438, cc1786-7. Disponible en: $\quad<$ https://api.parliament.uk/historic-hansard/commons/1947/jun/17/disabled-polesargentine>. Acesso em: 20 ago. 2020.

\section{FONDOS DOCUMENTALES DISPONIBLES EN LOS SIGUIENTES ARCHI-} VOS:

AKN. Archivo de Actas Nuevas, Varsovia, Polonia.

CEMLA. Centro de Estudios Migratorios Latinoamericanos. Cuenta con base de datos de ingresos de pasajeros en la República Argentina.

Lista Kryszta. <https://listakrzystka.pl/> Es una base de datos que reúne información sobre las personas polacas que participaron en la Fuerza Aérea durante la Segunda Guerra Mundial.

PIASA. Polish Institute of Arts and Sciences of America. Fondo: Legación Polaca en Río de Janeiro. Nueva York.

PISM: Polish Institute and Sikorski Museum. Londres. Base de datos de los soldados participantes.

TNA. Archivos Nacionales de Gran Bretaña. Colección "War Office”, Sección Polonia. Londres.

Biblioteca Nacional Mariano Moreno. Colección de Periódicos Polacos. Buenos Aires. 